\title{
An Unusual Complication of Gall Stones-Mirizzi Syndrome - Primary Care Perspective
}

\author{
Ahmed Rashid Shaik $^{*}$, Nirupama Keshavrao Patil ${ }^{2}$, Fathiya Muhammad Al-Meer ${ }^{3}$
}

${ }^{1}$ MBBS, MRCGP, DGM, Consultant Family Medicine, Primary Health Care Corporation, Al Mina Street (Second Ring Area Authority Opposite Double Tree Hilton Hotel، Doha, Qatar. P.O Box - 26555

${ }^{2}$ Consultant Radiologist, Primary Health Care Corporation, Al Mina Street (Second Ring Area Authority Opposite Double Tree Hilton Hotel، Doha, Qatar. P.O Box - 26555

${ }^{3}$ Senior Consultant Family medicine, Primary Health Care Corporation, Al Mina Street (Second Ring Area Authority Opposite Double Tree Hilton Hotel، Doha, Qatar. P.O Box - 26555

DOI: $10.36347 /$ sjmcr.2020.v08i12.002

| Received: 18.11 .2020 | Accepted: 30.11 .2020 | Published: 05.12.2020

*Corresponding author: Dr. Ahmed Rashid Shaik

Abstract

Mirizzi syndrome is a rare condition caused by the obstruction of the common bile duct or common hepatic duct and without preoperative diagnosis mortality could be as high as $17 \%$. It has been reported that $6 \%$ to $28 \%$ of patients with preoperative diagnosis of Mirizzi syndrome actually had gallbladder cancer. The disease can mimic cancer of the gallbladder, causing considerable diagnostic difficulties. In this current case A 22-year-old well gentleman presented with 4 days history of yellowish discoloration to eyes, skin, abdominal pain on eating food and weight loss of $10 \mathrm{kgs}$ over past 6 months. Icterus was note on examination. He was referred to secondary care for further evaluation. Blood test confirmed deranged Liver function tests. MRCP showed - Stones of the Extrahepatic bile ducts causing intrahepatic bile duct obstruction, probably located within the lower end of the anomalously crossing cystic duct causing extrinsic compression of the common duct (Merizzi Syndrome). A Laparoscopic Cholecystectomy + Trans cystic CBD exploration with Choledochoscope and extraction of stones by Dormia basket + Intraoperative Cholangiogram (IOC) was carried out. Findings were in keeping with Mirizzi syndrome. Blood tests done post operatively showed improvement in bilirubin and LFTs in span of 20 days. He was discharged home on oral antibiotics and analgesia. Mirizzi's syndrome is a rare complication caused by gallstone(s) located in the infundibulum of the gallbladder or cystic duct leading to adjacent biliary duct compression which results in partial or complete obstruction of the common hepatic duct and sometimes with the presence of cholecystocholedochal fistula. Incidence ranges from $1 \%$ per year in developed countries to upto $5.7 \%$ in developing countries. Surgical cholecystectomy is considered as the treatment of choice [3]. The surgeon is the main specialist for diagnosis and treatment but familiarity and a high degree of suspicion of this condition must be present by the initially treating primary caregiver or emergency physician. Delay in making an adequate diagnosis and treatment could lead to increased patient morbidity and mortality early recognition of jaundice and prompt referral to secondary care for further evaluation and management has been found to have favourable outcomes.

Keywords: Abdominal pain, Gallstones, Jaundice, mirizzi syndrome.

Copyright $\odot 2020$ The Author(s): This is an open-access article distributed under the terms of the Creative Commons Attribution 4.0 International License (CC BY-NC 4.0) which permits unrestricted use, distribution, and reproduction in any medium for non-commercial use provided the original author and source are credited.

\section{INTRODUCTION}

Most common cause of jaundice in primary care is biliary tree stones with a frequency of up to $33 \%$ [1]. Mirizzi syndrome is a rare condition caused by the obstruction of the common bile duct or common hepatic duct by external compression from multiple impacted gallstones or a single large impacted gallstone in Hartman's pouch. Mirizzi syndrome without preoperative diagnosis could be as high as 17\% [4]. Preoperative diagnosis of Mirizzi syndrome is difficult and can be made in only $8 \%$ to $62.5 \%$ of patients. It has been reported that $6 \%$ to $28 \%$ of patients with preoperative diagnosis of Mirizzi syndrome actually had gallbladder cancer [4]. Mirizzi syndrome is a condition difficult to diagnose and treat, representing a particular "challenge" for the biliary surgeon. The disease can mimic cancer of the gallbladder, causing considerable diagnostic difficulties [6].

\section{Case Report}

A 22-year-old well gentleman presented with 4 days history of yellowish discoloration to eyes and skin. Additionally, he had abdominal pain on eating food and weight loss of $10 \mathrm{kgs}$ over past 6 months. Initial 
systemic physical examination was unremarkable except for icterus. He was referred to secondary care for further evaluation and management.

Blood test confirmed deranged hyperbilirubinaemia of 262 (Direct 217), Alkaline Phosphatase 386, ALT 267, AST 132.

Abdominal ultrasound showed thickened walls of the gallbladder with a calculus suggestive of cholecystitis. MRCP showed - Stones of the Extrahepatic bile ducts causing intrahepatic bile duct obstruction, probably located within the lower end of the anomalously crossing cystic duct causing extrinsic compression of the common duct (Merizzi Syndrome)

A Laparoscopic Cholecystectomy + Trans cystic CBD exploration with Choledochoscope and extraction of stones by Dormia basket + Intraoperative Cholangiogram (IOC) was carried out. Findings were in keeping with Mirizzi syndrome with fistula communicating mid cystic to proximal CBD. IOC after the above procedure showed smooth passage of contrast to biliary tree. Blood tests done post operatively showed improvement in bilirubin (reducing to 125) and LFTs completely normalising within a span of 20 days. He was discharged home on oral antibiotics and analgesia.

\section{DISCUSSION}

Mirizzi's syndrome is a rare complication caused by gallstone(s) located in the infundibulum of the gallbladder or cystic duct leading to adjacent biliary duct compression which results in partial or complete obstruction of the common hepatic duct and sometimes with the presence of cholecystocholedochal fistula [2]. Incidence ranges from $1 \%$ per year in developed countries to upto $5.7 \%$ in developing countries. Most commonly presentations are with obstructive jaundice with right upper quadrant pain or dark urine. Endoscopic retrograde cholangiopancreatography is the best diagnostic modality which is an invasive procedure. Surgical cholecystectomy is considered as the treatment of choice [3].

\section{CONCLUSION}

A multispecialty approach must be taken for this condition. Accurate diagnosis with an accurate evaluation of the extent of this disease must be concluded by all involved. The surgeon is the main specialist for diagnosis and treatment but familiarity and a high degree of suspicion of this condition must be present by the initially treating primary caregiver or emergency physician. Delay in making an adequate diagnosis and treatment could lead to increased patient morbidity and mortality. Although definite cause of jaundice is difficult to be identified in the primary care, early recognition of jaundice and prompt referral to secondary care for further evaluation and management has been found to have favourable outcomes [5].

\section{REFERENCES}

1. Taylor A, Stapley S, Hamilton W. Jaundice in primary care: a cohort study of adults aged $>45$ years using electronic medical records. Family practice. 2012 Aug 1;29(4):416-20.

2. Fachrull F, Stephanie A, Mudjaddid E. Mirizzi's Syndrome. Indonesian Journal of Gastroenterology, Hepatology, and Digestive Endoscopy. 2015;16(3):190-3.

3. Valderrama-Treviño AI, Granados-Romero JJ, Espejel-Deloiza M, Chernitzky-Camaño J, Mera BB, Estrada-Mata AG, Ceballos-Villalva JC, Campos JA, Argüero-Sánchez R. Updates in Mirizzi syndrome. Hepatobiliary surgery and nutrition. 2017 Jun;6(3):170-178.

4. Beltrán M. A. (2012). Mirizzi syndrome: history, current knowledge and proposal of a simplified classification. World journal of gastroenterology, 18(34), 4639-4650. doi:10.3748/wjg.v18.i34.4639

5. Jones, M. W., \& Ferguson, T. (2019). Mirizzi Syndrome. [Updated 2019 Feb 22]. In: StatPearls [Internet]. Treasure Island (FL): StatPearls Publishing; 2019 Jan-. Available from: https://www.ncbi.nlm.nih.gov/books/NBK482491

6. Clemente G, Tringali A, De Rose AM, Panettieri E, Murazio M, Nuzzo G, Giuliante F. Mirizzi syndrome: diagnosis and management of a challenging biliary disease. Canadian Journal of Gastroenterology and Hepatology. 2018 Jan $1 ; 2018$. 